
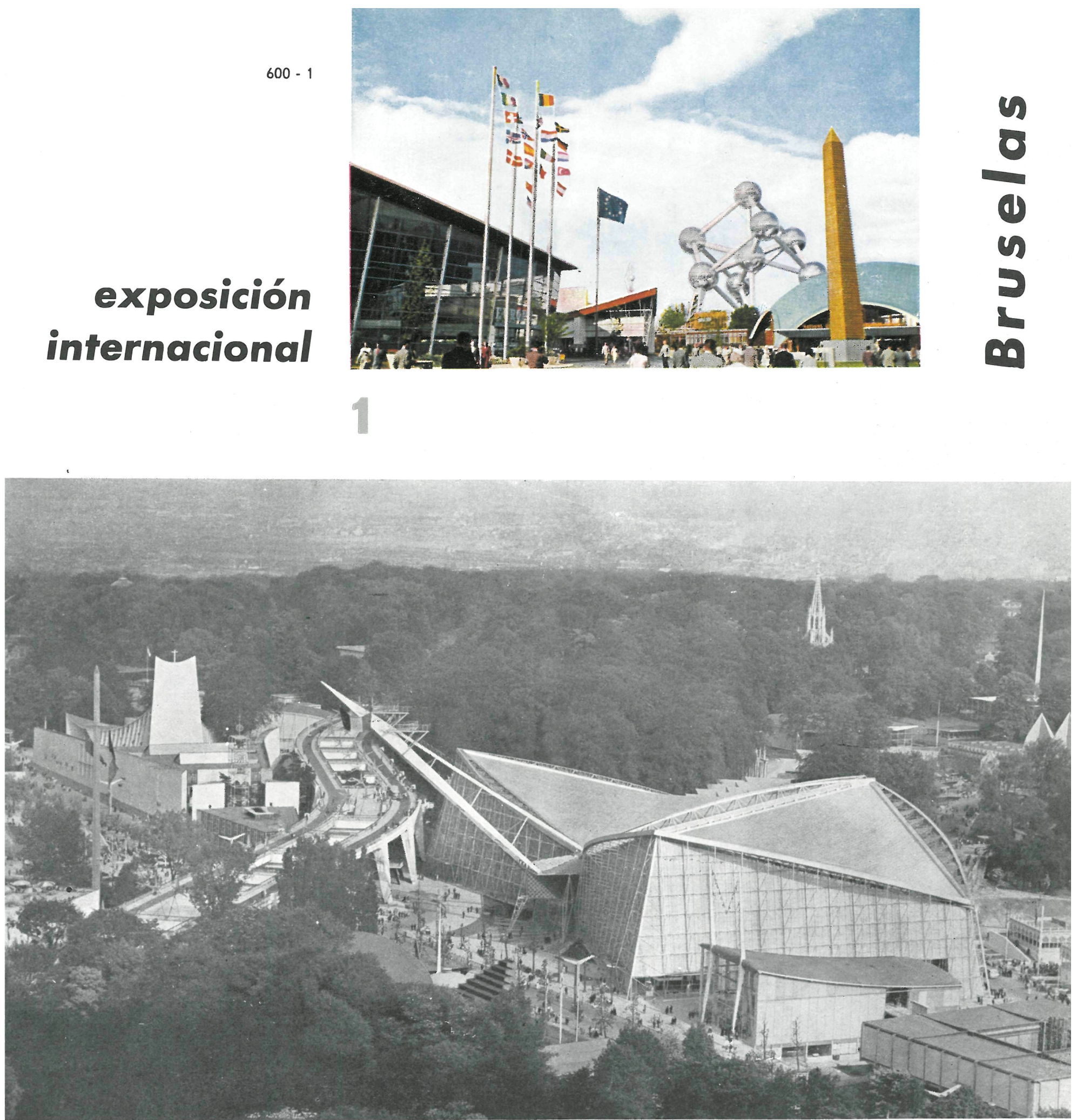

\title{
materiales de construcción
}

F. ARREDONDO, ingeniero de caminos

Se detallan los materiales de construcción más empleados en la Exposición de Bruselas, indicando las modalidades de uso de cada uno de ellos. Al lado de los materiales tradicionales se presentan materiales modernos, o de uso reciente, marcando tendencias para la industria de la construcción. 


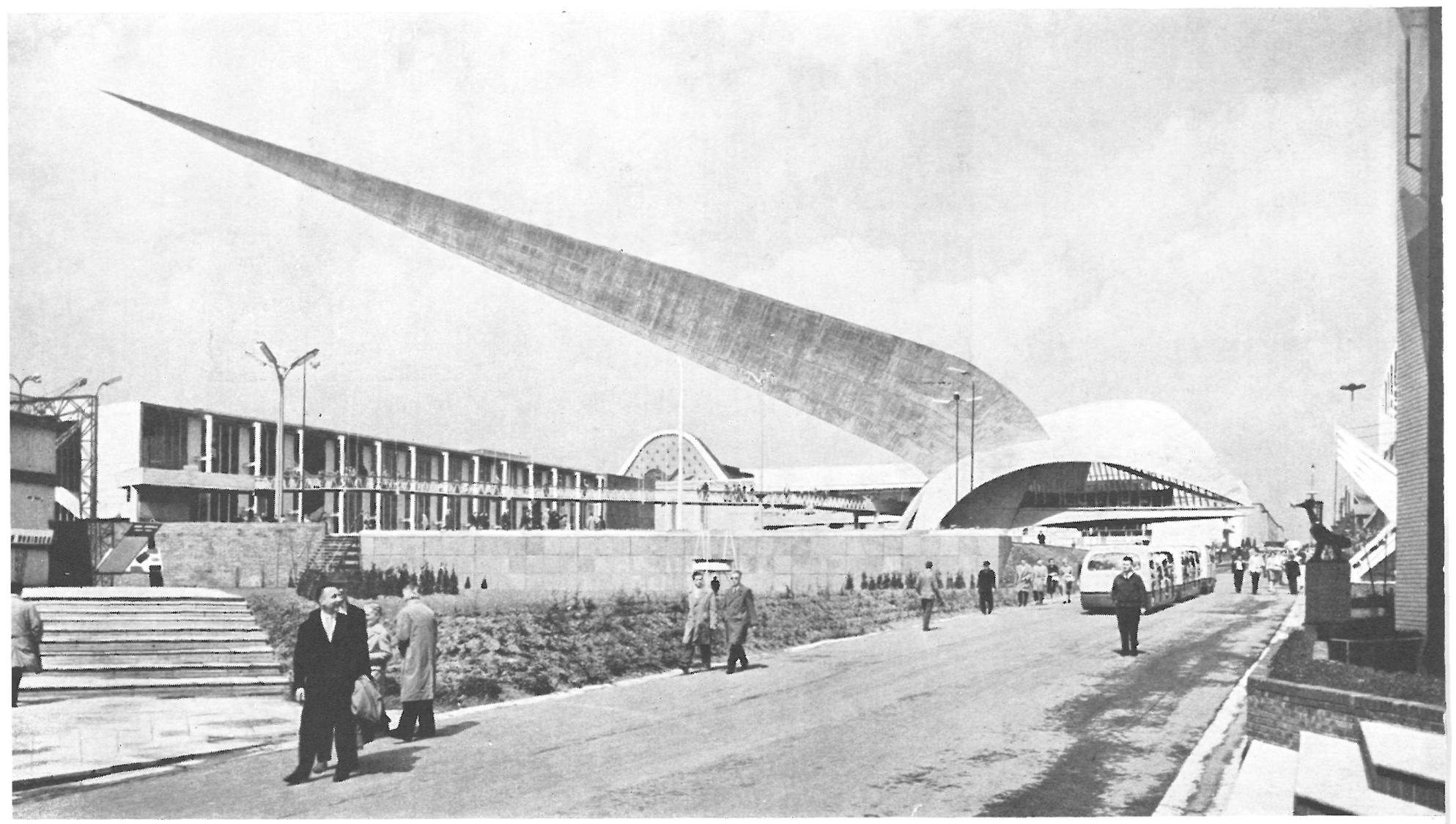

Es cierto que los pabellones de una exposición difieren bastante de la construcción en general.

La facilidad de montaje y desguace impone unas condiciones fácilmente cumplidas por las estructuras metálicas y cumplidas con menos facilidad por otros tipos de materiales.

Por otra parte, para la mayor vistosidad de los productos expuestos es de la mayor importancia conseguir una gran iluminación, tanto natural como artificial.

Pero independientemente de estas condiciones comunes a toda Exposición, no cabe duda que su conjunto representa unas tendencias y marca una tónica para la industria de la construcción.

En primer lugar, queremos destacar el profuso empleo del cristal. Unas veces se utiliza como elemento resistente; esto ocurre, por ejemplo, en el pabellón de Vidrio y Cerámica. A la entrada de este pabellón hay una pasarela sobre un foso, y el tablero de esta pasarela está formado por losas de cristal de unos $2 \mathrm{~cm}$ de espesor con una luz entre apoyos de $2 \mathrm{~m}$ aproximadamente.

Otras veces se utiliza el cristal para resolver el problema de la iluminación natural; esto sucede, por ejemplo, en el pabellón de la U. R. S. S., en el que todas sus fachadas son de cristal opaco, así como también gran parte del techo.

La figura 5 corresponde a este pabellón. Consta de una gran nave con la altura total en su parte central y con dos plantas en las zonas inmediatas a las fachadas. Desde distintos puntos de esta segunda planta hemos podido obtener fotografías del interior sin flash y sin exposición, lo cual da una buena idea de la luz que existe en su interior.

El acero juega un papel importante en esta Exposición. Existen muchos pabellones construídos con este metal, tal vez por la facilidad de montaje y desguace a que antes hemos aludido.

Por ejemplo, el pabellón de Francia tiene una estructura metálica con unos nudos enormes en su interior (figura 2). La cubierta está formada por dos paraboloides hiperbólicos con generatrices rectas constituídas por cables de acero.

Son varias las cubiertas de cables que pueden verse en Bruselas. Entre otros pabellones con cubiertas de este tipo están: el de Brasil, el de Estados Unidos y el de la Comunidad Europea del Carbón y el Acero.

También los pabellones de Checoeslovaquia, de España y parte del de Japón son de estructura metálica. Los pabellones de Austria y Alemania tienen estructura metálica vista, y este último es de una diafanidad grande en todas sus diferentes secciones.

Al hablar del acero no resistimos la tentación de mencionar el célebre Atómium, formado por nueve esferas de $18 \mathrm{~m}$ de diámetro, cada una de las cuales está constituída por doce elementos semicirculares de acero soldado. 

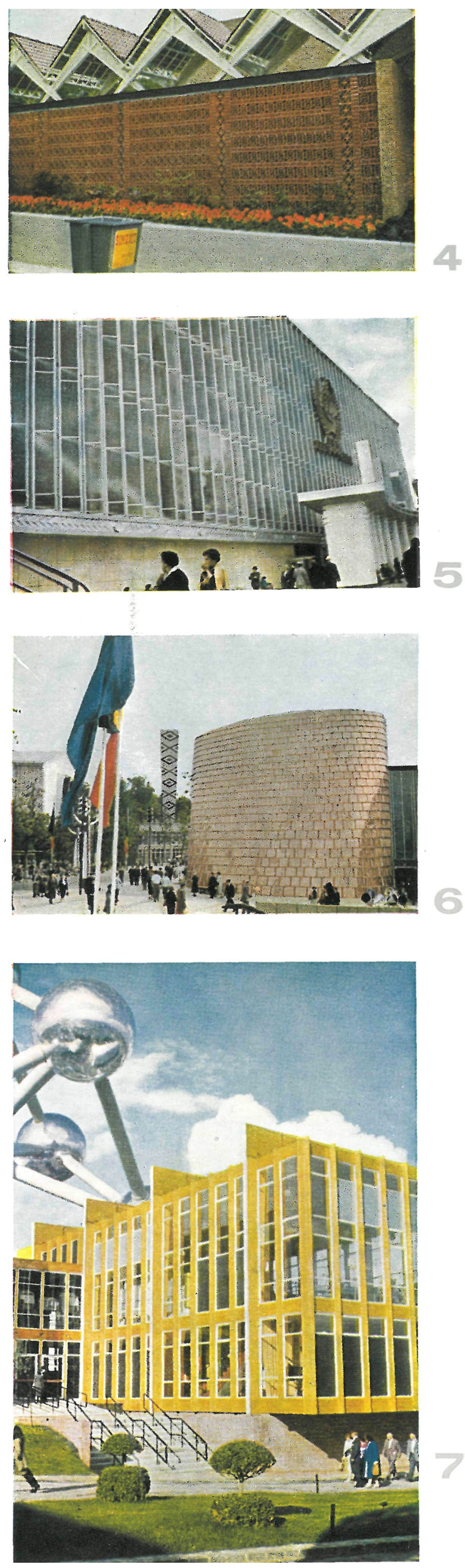

Estas esferas están unidas por tubos de $3,30 \mathrm{~m}$ de diámetro y $30 \mathrm{~m}$ de longitud. La altura total es de $100 \mathrm{~m}$ y su peso de 2.300 toneladas métricas (fig. 1).

Las esferas están forradas exteriormente por placas de aluminio brillante de $1,2 \mathrm{~mm}$ de espesor.

El aluminio tiene una gran intervención en todos estos edificios que comentamos. Raro es el pabellón que no tiene algo de aluminio, y algunos mucho, como el pabellón de Rusia, en el que, aunque la estructura es de acero, las fachadas son de aluminio y cristal (fig. 5).

Pero el aluminio no se utiliza sólo en elementos estructurales - en carpintería metálica, sino que también se emplea en grandes paneles de cerramiento, y con esta misión específica.

También hemos visto como revestimiento, y en un alarde de decoración que no debe ser barato, el chapado de cobre (fig. 6).

El hormigón también está representado en esta Exposición, pero, salvo en algunas construcciones determinadas, no se hace fácilmente visible. Indudablemente las cimentaciones son de hormigón, y algunas tienen una gran importancia. La del Atómium, por ejemplo, consta de 123 pilotes de unos $17 \mathrm{~m}$ cada uno: 59 de ellos están bajo la esfera inferior, y los restantes bajo las sustentaciones bípodas. Las cabezas de los 59 centrales están unidas por una placa de hormigón armado, de $12 \mathrm{~m}$ de diámetro y $2 \mathrm{~m}$ de altura.

Pero, aparte de esto, hay notables representaciones de este material y vamos a referirnos a tres de ellas:

Una de ellas, que salta a la vista en cuanto se entra a la Exposición por la puerta de la Cooperación Universal, es la cubierta del pabellón de la O. N. U. (fig. 1). Es un casquete esférico apoyado en seis puntos. Está realizado en hormigón armado de $8 \mathrm{~cm}$ de espesor.

Otra de las representaciones masivas del hormigón es una pa sarela elevada, de unos $400 \mathrm{~m}$ de longitud, que salva una depresión en el parque, y desde la cual, debido a su elevación máxima de $15 \mathrm{~m}$ sobre el suelo, se pueden ver magníficos panoramas de la Exposición (figs. 2 y 8).

Consta de un tablero soportado por 124 pórticos de hormigón pretensado. Según tenemos entendido, el peso de estos pórticos es de 25 toneladas cada uno, y fueron prefabricados en Brujas y trasladados después al punto de emplazamiento.

En el pabellón de la Ingeniería civil belga hay un elemento de hormigón armado, que consiste en una estructura en forma de flecha apoyada en un extremo y libre en el otro; tiene $85 \mathrm{~m}$ de longitud y su extremo libre está a $35 \mathrm{~m}$ del suelo (fig. 3).

Su sección transversal tiene forma de A invertida y está prorectada, según creemos, como viga de igual resistencia con espesores que varían de 4 a 12 centímetros.

Esta flecha soporta una pasarela sobre un plano de Bélgica y al mismo nivel de la pasarela, es decir, a $5 \mathrm{~m}$ sobre el suelo, hay una sala triangular, de $28 \mathrm{~m}$ de lado, cuyo suelo es una losa de hormigón armado y cuya cubierta es una membrana del mismo material, de $6 \mathrm{~cm}$ de espesor mínimo, que se prolonga, hasta el suelo, en dos puntales, los cuales, con el apoyo central, forman un trípode que contribuye a dar estabilidad transversal al conjunto.

La cerámica casi no existe. Aparte de algunos exteriores en el pabellón de España, hemos encontrado toda la decoración interior del pabellón de Italia realizada en un ladrillo tosco con llaga rehundida.

En el pabellón de Vidrio y Cerámica existe un vistoso cerramiento construído con bloques para forjados (fig. 4), y otro análogo en el pabellón de Estados Unidos para alojar la sala del circarama.

La madera tiene una representación lucida. En algunos pabellones, por ejemplo, el de Alemania, todo el suelo es de madera, con un barniz sintético protector.

Las maderas laminadas y encoladas tienen aceptación, y puede verse su uso en el pabellón dedicado a estudios de productividad. planeamiento y control (fig. 7), en una pasarela que da paso al parque de atracciones y en otras varias construcciones. 


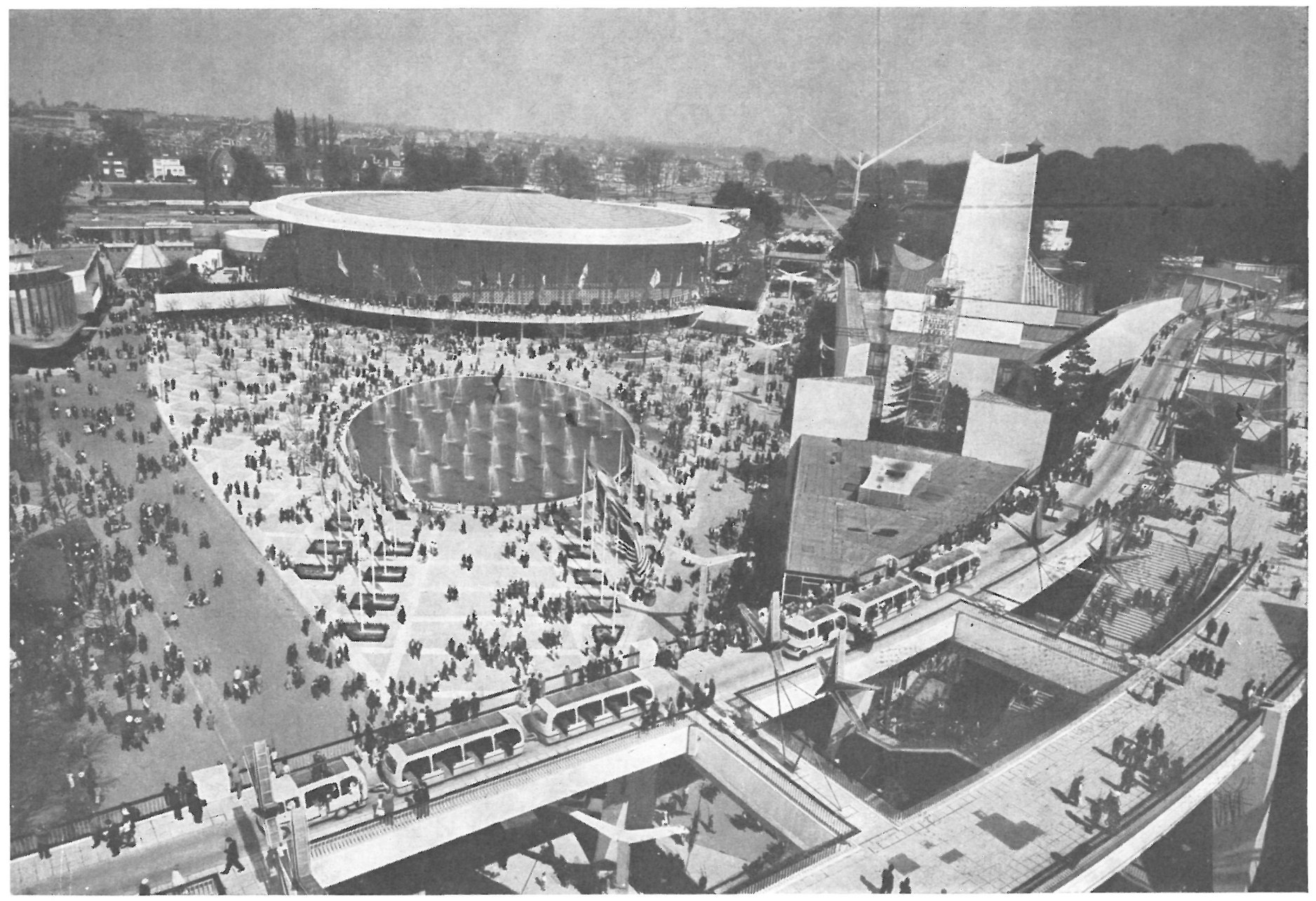

Estas maderas llevan un tratamiento superficial que las preserva de la intemperie.

Como es natural, los pabellones de los países nórdicos están constituídos enteramente de madera.

También el pabellón del gobierno inglés es de madera. Tiene una forma caprichosa que recuerda la de tres cristales mineralógicos unidos. Cada "cristal" está constituído por ocho elementos triangulares de madera contrachapada, hechos con el mismo principio de las alas de los aviones. En total tienen 6,5" de grueso (dos tableros laterales de $1 / 4$ de pulgada y 6 " de espaciadores intermedios).

Estos elementos fueron fabricados en Inglaterra y montados en la Exposición sin más necesidad que una grúa. No llevan ninguna armadura interior y van provistos de pintura protectora contra la intemperie y el fuego.

Existen muchos pabellones en cuya construcción o decoración se utilizan los materiales plásticos. Destaca por su importancia y magnitud el pabellón norteamericano, con estructura metálica y un cerramiento exterior de plástico detrás de una celosía también metálica (fig. 8).

Aparte de esto, el uso de los plásticos está enormemente extendido para solados. En numerosos pabellones hemos encontrado suelos de este tipo, algunos continuos, pero en la mayoría en losetas. Algunos deben tener debajo goma-espuma o cosa análoga que produce una grata sensación.

Hay además tres elementos, que si bien no se pueden considerar materiales de construcción, contribuyen de forma extraordinaria a la decoración de la Exposición:

Uno de ellos es el agua que, en diversos juegos, surtidores y cascadas, produce un efecto agradable y presta su concurso a la belleza del conjunto.

Otro de estos elementos es la luz. La catarata de luces de todos los colores hace que la vistosidad de la Exposición no se pierda al ponerse el sol y origina un sorprendente e impresionante aspecto nocturno.

El tercer factor que colabora de modo notable a la grandiosidad de este certamen y que pone un soberbio marco a cuanto en él se expone, es el magnífico parque en que están situados los pabellones de las diversas naciones. Tal vez se acusa más en esta parte de la Exposición la existencia de las masas verdes, por contraste con la zona belga, casi exenta de arbolado. 